$20 \mid 2015$

NOVECENTO... E DINTORNI

Foscolo e la cultura europea

\title{
Foscolo in Polonia tra Otto e Novecento
}

Foscolo en Pologne entre XIX et XX siècle

Foscolo in Poland between 19th and 20th Century

Anna Tylusińska-Kowalska

\section{(2) OpenEdition}

Journals

Edizione digitale

URL: http://journals.openedition.org/cei/2497

DOI: 10.4000/cei.2497

ISSN: 2260-779X

Editore

UGA Éditions/Université Grenoble Alpes

\section{Edizione cartacea}

Data di pubblicazione: 30 juin 2015

Paginazione: 189-200

ISBN: 978-2-84310-289-9

ISSN: 1770-9571

Notizia bibliografica digitale

Anna Tylusińska-Kowalska, «Foscolo in Polonia tra Otto e Novecento», Cahiers d'études italiennes

[Online], 20 | 2015, online dal 01 janvier 2017, consultato il 26 mars 2021. URL: http://

journals.openedition.org/cei/2497 ; DOI: https://doi.org/10.4000/cei.2497 


\title{
FOSCOLO IN POLONIA TRA OTTO E NOVECENTO
}

\author{
Anna Tylusińska-Kowalska \\ Uniwersytet Warszawski
}

\section{Echi foscoliani nei capolavori del Romanticismo polacco}

La storia della ricezione di Foscolo in Polonia tra Otto e Novecento - la storia della sua 'fortuna' o 'sfortuna' polacca - può essere divisa in alcune fasi. In questa sede cercheremo di mostrare come la fortuna del grande poeta italiano sia passata da una stagione in cui era quasi sconosciuto (la prima metà dell'Ottocento), a un periodo in cui nacque un certo interesse per la sua opera (la seconda metà dell'Ottocento), a un'epoca in cui cominciò a essere tradotto e commentato (gli anni Venti e Trenta del Novecento). Fino alla traduzione quasi integrale e molto più seria del 1979.

Possiamo supporre che Foscolo, ignorato dalla critica letteraria polacca del primo Ottocento, fosse invece conosciuto da grandi poeti come Mickiewicz o Słowacki, nelle cui opere si sentono echi foscoliani più o meno lontani, soprattutto dell'Ortis. Nella seconda metà dell'Ottocento l'opera di Foscolo è invece ricordata e anche brevemente analizzata negli studi del grande storico della letteratura Henryk Lewestam. Verso la fine del secolo, nel I885, è pubblicata la prima traduzione in polacco delle Ultime lettere, importante sia per il suo valore informativo che letterario, e che paragoneremo in seguito a quella del 1979. L'interesse per il poeta cresce poi negli anni compresi tra le due guerre.

La Polonia subì tre spartizioni negli ultimi decenni del Settecento e dopo l'ultima, avvenuta nel I795, sparì dalle cartine geografiche d'Europa. Il suo territorio fu diviso tra le potenze confinanti e la vita culturale del paese fu sottoposta all'occhio vigile della censura, che spietatamente eliminava ogni traccia di pensiero liberale, sia per quanto riguarda la stampa periodica che per la produzione letteraria e le scene dei teatri.

In queste circostanze i nostri grandi poeti romantici - Mickiewicz, Słowacki, Krasiński - presero la via dell'esilio e i loro capolavori nacquero 
tra Parigi, Roma e Napoli. Tutti e tre risiedettero a lungo in Italia, dove respirarono non solo l'aria del Mediterraneo, ma anche quella della letteratura e della cultura del tempo. Mickiewicz, come noto, ebbe stretti legami sia con Mazzini che con Tommaseo e s'impregnò dell'atmosfera post-napoleonica. Come dirà più tardi Julia Wieleżyńska, Gustaw, il protagonista del suo grande poema drammatico Gli Avi, è una specie di fratello minore di Jacopo. Il poema fu sottoposto a uno studio approfondito da parte di Carlo Cattaneo che lo pubblicò sul «Politecnico» nel I86I ${ }^{\mathrm{I}}$.

Non meno interessante è il caso di Juliusz Słowacki (I809-I849), autore di drammi e poemi di forte impronta patriottica, anima irrequieta e sensibilissima, tormentata da contraddizioni ideologiche e psicologiche, che con il suo vagare continuo tra vari paesi d'Europa ci ricorda la biografia e il carattere di Ugo Foscolo. Il suo dramma romantico Kordian, ritenuto il suo capolavoro, non fu molto noto all'inizio, e fu apprezzato solo da qualche critico letterario polacco. Non è mai stato tradotto in italiano. Si tratta di un testo molto particolare, caratterizzato da spunti mistici, un forte simbolismo politico e molta couleur locale, per cui poco si presta a un'analisi spassionata e razionale. Il suo messaggio complesso e non sempre decifrabile è stato studiato recentemente in Italia da un'agguerrita nuova generazione di polonisti.

Il dramma vide la luce a Parigi nel I835. Il nome del protagonista allude al suo 'cuore', cioè ai suoi forti affetti, proprio come il nome di Jacopo Ortis alludeva a Jean-Jacques. Ma i tempi sono diversi, un'intera generazione separa Słowacki da Foscolo. Kordian, come altri eroi romantici polacchi, è vittima del mal du siècle: inetto, idealista, incapace di ambientarsi nella realtà che lo circonda, vorrebbe contribuire alla liberazione della patria, ma è passivo e nevrotico. Disprezza la gente che lo circonda, sembra pure disprezzare se stesso, ma è pronto a compiere atti eroici che descrive in lunghissimi monologhi.

Per fare i conti con la realtà e con se stesso, e dare espressione alla sua umanità, Kordian sceglie i luoghi più appartati e selvaggi, fra cui la vetta del Monte Bianco, ma il suo impegno patriottico rimane un'esperienza solo affettiva, e richiama vagamente i monologhi di Jacopo sui Colli Euganei. Crollate le sue speranze (il complotto contro lo zar fallisce, i cospiratori vengono denunciati e condannati), reduce inoltre da una cocente delusione amorosa, tenta il suicidio. Ma a differenza di Ortis, che è disperato,

I. Cfr. A. Tylusińska-Kowalska, Carlo Cattaneo, precursore del positivismo?, in F. Coniglione (a cura di), Il positivismo italiano: una questione chiusa?, Atti del Congresso di Catania (I3-I4 settembre, 2007), AcirealeRoma, Bonanno, 2008, pp. 633-643. 
ma capace di compiere il gesto fatale, Kordian non riesce né a suicidarsi né a uccidere il governatore della Polonia Kostantin, fratello dello zar Nicola I. Scoperto il complotto, il giovane è rinchiuso in manicomio.

Kordian presenta alcune affinità di carattere con l'Ortis: come lui viaggia molto durante la giovinezza, e i suoi sono gli spostamenti continui di un nevrotico che fugge dal proprio 'io', che vaga - come Foscolo attraverso l'Europa ma ovunque incontra solo delusioni. I brani in cui parla del Papa mostrano un atteggiamento pieno di conflitti interni e contraddizioni ${ }^{2}$. La figura di Laura - baciata in una scena paragonabile a quella dell'Ortis — fa pensare a quella di Teresa nell'Ortis: idealizzata, angelicata dal poeta, porta il nome della musa di Petrarca.

Si dirà forse che le affinità tra le due opere sono conseguenza del clima generale - culturale, letterario e politico - dell'epoca. Foscolo (impregnato di filosofia illuminista), e Słowacki (pervaso di una religiosità romantica molto personale), non hanno forse niente in comune. Ma a Parigi, dove i polacchi s'incontravano regolarmente con gli esuli italiani (tra cui Niccolò Tommaseo, Terenzio Mamiani e altri), si parlava di politica e della situazione in cui versavano le due nazioni. Foscolo, grazie all'Ortis, era considerato un ispiratore e un maestro. Sembra quindi legittimo accostare il suo capolavoro a quello di Słowacki.

\section{Fortuna di Foscolo nella critica letteraria polacca dell'Ottocento}

Nella prima metà dell'Ottocento Foscolo è menzionato solo due volte dalla critica letteraria polacca. La prima volta nel saggio del I 842 di Henryk Lewestam (I822-I878) Literatura dramatyczna we Wtoszech [La letteratura drammatica in Italia] uscito a Varsavia nella rivista da lui fondata: «Roczniki Krytyki Literackiej». In un passo dedicato ad Alfieri leggiamo che «Monti e Foscolo che, dopo l'Alfieri, arricchirono la scena italiana di opere proprie non possono essere considerati che suoi discepoli o imitatori, mai elevatisi all'altezza del maestro» ${ }^{3}$. Alfieri era molto stimato in Polonia, accanto a lui Foscolo rimaneva nell'ombra.

2. Nella Polonia cattolicissima le autorità avrebbero censurato una polemica contro Gregorio XVI, ma Kordian fu per la prima volta pubblicato a Parigi. Ricordiamo che Niccolò Tommaseo fu più coraggioso, e lanciò la sua invettiva contro il Pontefice, nel suo testo politico-religioso Dell'Italia, anch'esso dato alle stampe a Parigi.

3. H. Lewestam, Wtoska literatura dramatyczna [La letteratura drammatica in Italia], "Roczniki Krytyki Literackiej", I842, $\mathrm{n}^{\circ}$ Io, p. 38. 
Una riflessione su Foscolo ci è proposta anche da Edward Dembowski (I822-I846), leader e dittatore della rivoluzione di Cracovia del I846, la cui personalità potrebbe essere accostata a quella di Mazzini in quanto, oltre all'impegno politico, fu un critico letterario 'militante', protagonista di numerose polemiche. In un saggio del I843, intitolato Piśmienność wspótczesna [Letteratura contemporanea] e apparso sul "Przegląd Naukowy» chiama Foscolo e Pellico autori 'geniali', poi però aggiunge: «Foscolo fu solo capace di esprimere il suo odio verso il mondo in forma elegante. Alfieri fu la luce, gli altri scrittori [Foscolo e Niccolini] la sua aureola» ${ }^{4}$. Il giudizio molto positivo su Pellico non può stupirci perché era l'autore più letto, più tradotto e più amato dai critici polacchi dell'Ottocento.

Un primo tentativo di far conoscere Foscolo al pubblico polacco si ebbe con l'Enciclopedia universale e con la Storia della letteratura universale, due opere degli anni Sessanta dell'Ottocento. Nel volume 9 dell'Enciclopedia, Lewestam dedica al poeta italiano un'intera pagina'. Inizialmente, il filo conduttore del ragionamento è costituito dal patriottismo di Foscolo (presentato come: Mikołaj Hugo) e il lettore polacco è informato che tutta la vita e l'opera del poeta furono dedicate alla patria. Plasmato dagli ideali della Rivoluzione francese, aveva visto come altri in Napoleone il liberatore d'Italia e aveva cambiato atteggiamento solo dopo il trattato di Campoformio. Lewestam trova nel Tieste le tracce di un forte impegno politico, mentre la delusione per la cessione di Venezia all'Austria troverà espressione nelle Ultime lettere di Jacopo Ortis.

Sempre secondo Lewestam (male informato su questo punto), Foscolo fu invitato ai famosi comizi di Lione e vi avrebbe pronunciato un'orazione che aveva per scopo di indurre Napoleone a concedere agli italiani una costituzione democratica, rispettosa dei loro diritti. Il suo discorso sarebbe durato parecchie ore e avrebbe riscosso applausi scroscianti. Esatta invece l'informazione sulla pubblicazione postuma dell' Orazione, nel I829 a Lugano. Il critico ci informa anche dell'insegnamento all'Università di Pavia, ma sostiene che dopo l'Orazione inaugurale la censura avrebbe soppresso le successive lezioni. Lewestam ci mostra poi il poeta come ufficiale dell'esercito francese a Boulogne, ma questa parentesi militare fu di breve durata, poi Foscolo si stabilì a Milano e pubblicò Dei Sepolcri in cui la

4. E. Dembowski, Piśmienność powszechna [Letteratura universale], Pisma, PWN, Warszawa, I955 [ristampa da «Przegląd Naukowy», I843, nº 29], vol. IV, pp. II64-II77.

5. Cfr. la voce Foscolo (a cura di H. Lewestam) in Encyklopedya Powszechna [Enciclopedia universale], nakł. S. Olgerbranda, Warszawa, vol. IX, I864, p. 98. 
saggezza antica si fonde con il pensiero moderno. Nella stessa città mise in scena l'Aiace che per via delle allusioni troppo esplicite a Napoleone gli valse l'esilio. Si recò allora a Firenze dove scrisse un'altra tragedia, Ricciarda, pubblicata a Londra nel I820, e in cui espresse speranza che l'unità d'Italia fosse imminente. Perdoniamo al critico polacco che forza qui il testo.

Lewestam fornisce importanti dati biografici: ricorda la collaborazione del poeta all'Edimburgh Review dove pubblicò saggi di altissimo livello su Dante, Boccaccio e Petrarca. Indica che per l'edizione dantesca ricevette il compenso di $16000 \mathrm{zl}$. Sostiene che Foscolo sarebbe stato il primo vero studioso di Dante. La voce si chiude con i titoli (in italiano) delle ultime pubblicazioni foscoliane e della biografia di Pecchio: Vida [sic] di Ugo Foscolo (Lugano, I833). In compenso, nel volume 27 della medesima Enciclopedia universale, alla voce "Letteratura italiana", nelle pagine dedicate alla letteratura contemporanea, sono elencate le opere di Pindemonte, Alfieri e Monti, ma il nome di Ugo Foscolo non compare neppure una volta.

Nella Storia della letteratura universale Lewestam apre la pagina dedicata a Foscolo con le seguenti parole: "Alla scuola di [Alfieri] appartiene in primo luogo, simile a lui anche per carattere, Foscolo. Odiando sinceramente sia gli Austriaci che i Francesi fu costretto a transcorrere una parte della sua vita in esilio» ${ }^{6}$. Seguono brevi informazioni biografiche. Del Tieste è detto che fu scritto sulle orme di Alfieri. Ma a Lewestam piace più di tutte la Ricciarda. Egli ricorda che l'opera di maggior successo fu l'Ortis, definito «romanzo politico-fantastico-sentimentale», poi riporta (in italiano!) i titoli dei saggi critici su Decamerone e Dante, ma in inglese quello del saggio su Petrarca.

La nota critica si chiude con l'elogio delle opere poetiche di Foscolo, Dei sepolcri, La chioma di Berenice e Le Grazie, presentate come un Inno alle Grazie. Il tutto nel quadro di un'Introduzione alla letteratura italiana dell'Ottocento in cui Lewestam punta sul registro politico-patriottico, di cui dice che influì profondamente sul modo di scrivere, e contribuì alla rinascita della lingua italiana, fino ad allora condizionata fortemente dalla moda francese. Lewestam si rallegra che l'Italia abbia realizzato la propria unità ed è convinto che d'ora in poi la sua letteratura continuerà a svilupparsi in modo autonomo.

6. H. Lewestam, Historya literatury powszechnej [Storia della letteratura universale], nakł. A. Lewińskiego, Warszawa, 1865 , p. 356. 


\section{Le due traduzioni delle Ultime lettere}

Nel I885, le Ultime lettere furono per la prima volta pubblicate in traduzione polacca ad opera di Adam Grąbczewski, noto pubblicista e critico, collaboratore alle più importanti riviste letterarie polacche dell'epoca7. Bisognerà aspettare quasi un secolo per averne una nuova.

La traduzione omette l'avviso al lettore dell'amico Lorenzo, reintrodotto invece in quella più recente di Barbara Sieroszewska, e quindi sopprime anche i suoi successivi interventi in prima persona. Ne risulta un testo molto emotivo e patetico, in cui le frasi più lunghe sono impietosamente tagliate dal traduttore, insomma, una traduzione parziale, che riduce di molto le dimensioni del testo e ne offre un'interpretazione superficiale.

Paragoniamo in particolare l'epilogo del romanzo nelle due traduzioni. Nella versione del I885, assistiamo a due scene parallele: da un lato l'agonia di Jacopo in presenza del signor T., che fa di tutto per salvarlo bloccando l'emorragia, dall'altro il viaggio di Lorenzo che appena arrivato irrompe nella stanza dell'amico, ma lo trova ormai privo di vita. L'ultima frase, che richiama l'atmosfera dei romanzi gotici ${ }^{8}$, è di Lorenzo, ma il lettore polacco non sa bene chi sia e perché se lo trovi all'improvviso davanti.

Entrambi i traduttori si collocano a metà strada fra il registro poliziesco e quello romantico e ricorrono a sostantivi e aggettivi che appartengono agli stessi campi semantici: 'sangue', 'morte' (declinata in vari modi), 'disperazione', 'dolore', 'cuore', 'agonia', 'cadavere'. Ma nel complesso la traduzione di Sieroszewska è molto più fedele, riproduce esattamente l'ultima pagina del romanzo e esprime in modo suggestivo lo stato d'animo di Teresa? ${ }^{9}$.

A parte il lessico più pacato e più piatto, la Sieroszewska ricorre alle note per spiegare ai lettori polacchi nomi di luogo e eventi storici, come il trattato di Campoformio. Negli anni della sua traduzione la Polonia era comunista e spesso guardava all'Italia come a un modello, non esitando a importarne la letteratura, soprattutto testi storici d'impianto patriottico-martirologico. Si

7. H. Foscolo, Listy Jakóba Ortisa, nakł. księgarni K. Łukaszewicza, Warszawa, I885.

8. «W lewej piesi tkwił sztylet. P.T. odkrywszy bieliznę usiłował zatamować upływ krwi — Jakób zlekka uścisnął dłoń jego, głowa spadła na poduszkę i skonał. Pospieszyłem z przybyciem mojem do Padwy i wpadłszy do mieszkania Jakóba zastałem ojca Teresy, chirurga i plebana. Nie wiem, zkąd starczyło mi sił na zniesienie tak bolesnego widoku. Położyłem rękę na jego sercu — już nie drgało. Teresa pogrążyła się w niemej boleści. W nocy pochowaliśmy go na wzgórzu pomiędzy jodłami» (ivi, p. I86).

9. «Nie wiem, skąd wziąłem tyle siły, żeby zbliżyć się i położyć rękę na piersi Jacopa, tuż obok rany. Był martwy, już zimny. Nie płakałem, nie mogłem wydobyć głosu. Stałem, otępiałym wzrokiem wpatrując się w tę krew, dopóki nie przyszedł proboszcz, a zaraz po nim chirurg; obaj z pomocą domowników oderwali nas przemocą od tego straszliwego widoku. Teresa przez wszystkie te dni trwała pogrążona w żałobie, w śmiertelnym milczeniu. W nocy powlokłem się za zwłokami, które trzech wieśniaków pogrzebało na szczycie wzgórza, pod piniami» (U. Foscolo, Ostatnie listy Jacopa Ortis, trad. B. Sieroszewska, Czytelnik, Warszawa, 1979, p. 165). 
deve comunque dire che tutta la letteratura classica straniera era pubblicata in Polonia, sia pure in tirature ridotte, cosa che non succedeva in altri paesi del blocco sovietico, come la DDR.

La traduttrice ha contribuito molto alla divulgazione della letteratura italiana in Polonia, traducendo fra l'altro Il Gattopardo di Tomasi di Lampedusa. Il romanzo di Foscolo è seguito da una postfazione, necessaria per presentare al pubblico degli anni Settanta un autore che rimaneva sconosciuto in Polonia, e di cui riproduco qui, a titolo indicativo, le ultime parole:

Il romanzo giovanile del nostro poeta racconta la storia di illusioni nobili e commoventi, non è una copia senz'anima della realtà, tiene conto delle emozioni e degli avvenimenti contemporanei. Ė documento interessante di un'epoca tragica e perciò il grido d'amore, il patriottismo e la disperazione possono interessare anche il lettore di oggi ${ }^{10}$.

\section{Ugo Foscolo nella critica letteraria polacca del periodo interbellico}

Per farsi un'idea più precisa della fortuna/sfortuna di Foscolo in Polonia, è utile gettare uno sguardo su alcuni saggi usciti nei primi tre decenni del Novecento, periodo che segna una svolta nell'approccio a Foscolo. Essi dimostrano l'emergenza di un interesse per poeti come Foscolo o Leopardi, che nell'Ottocento erano quasi sconosciuti, e non sufficientemente apprezzati. Grazie ad alcuni notevoli italianisti del periodo interbellico, furono introdotte in Polonia la grande prosa e la grande poesia italiana dell'Ottocento.

Nel 1909 il «Kurier Pomorski» pubblica un lungo testo su Leopardi del noto pubblicista e critico letterario Antoni Szowacki che in un'analisi delle canzoni patriottiche di Leopardi elenca i poeti cui si sarebbe ispirato il recanatese, e fra questi ricorda Ugo Foscolo. Fra le fonti di Leopardi vi sarebbero alcune opere foscoliane, fra cui l'Ortis, definito «romanzo psicologico», e «il grande Werther». L'Ortis è citato in particolare per il brano della lettera di Ventimiglia che comincia con le parole: «I tuoi confini, o Italia, son questi!» e che è tradotto in modo non del tutto fedele, certo adattando il testo ai bisogni ideologici del pubblicista, ma senza distorcerne del tutto il pensiero, di cui è sottolineata la profondità ${ }^{\text {II }}$. Invece di puntare sulla lucidità del pensiero del Foscolo, Snowacki ne dilata le

Iо. Ivi, p. I9I.

II. A. Snowacki, Tto historyczne i geneza kancon patryotycznych Giacomo'a Leopardiego [Lo sfondo storico e la genesi delle canzoni patriottiche di Giacomo Leopardi], «Kurier Poznański», Poznań, I909, n I29, p. 2. 
affermazioni patriottiche e fa dell'appello alla concordia un'esortazione all'unità politica. In quegli anni, l'interesse per la cultura e la letteratura italiana è strettamente legato alla situazione politica dei due paesi, l'Italia suscita notevole solidarietà e simpatia, e l'autore più amato dai polacchi è Pirandello, che essi sentono vicino per le sue incertezze e il suo relativismo. E tuttavia anche Foscolo e Leopardi suscitano, come si è detto, un certo interesse.

Il 20 aprile del 1924, durante la seduta della Società dei Filosofi, il prof. Tadeusz Zieliński lesse una lunga relazione, un vero e proprio studio su Foscolo. Su di essa si basò il discorso critico originale di una grande italianista, laureatasi a Roma, e traduttrice da ben I2 lingue: Julia Wieleżyńska. Lo studio s'intitolava: Ugo Foscolo, uomo, letterato, critico, e la rivista della Società dei Filosofi ne ospitò una versione abbreviata, destinata a fare da prefazione a una traduzione delle poesie di Foscolo che alla fine rimase però inedita.

Il saggio muove dall'osservazione che l'attività politica e la produzione poetica di Foscolo si svolse in un'epoca in cui l'Italia cominciava lentamente a svegliarsi, sul piano letterario e morale, dal letargo del Settecento. Oltre al talento, gli scrittori che sorgono in quel momento - democratici e patrioti - possiedono una qualità dimenticata da tanto tempo: la coscienza italiana. E sarà questo il denominatore comune della letteratura italiana dell'epoca. Parini diede una scossa alla nazione con le sue satire, Alfieri la educò col teatro. Foscolo si nascose dietro all'uno e all'altro considerandoli suoi modelli: «Nella ricostruzione appena iniziata dell'anima nazionale egli fu il terzo», constata la Wieleżyńska.

La studiosa commenta subito dopo alcuni segmenti della biografia del Foscolo:

Le opere del soldato, una vita piena di stenti e di penuria in esilio, azioni compiute controvoglia (come quella di collaborare con Napoleone). Di carattere passionale, fuggiva dalla quotidianità spesso squallida nella poesia. Sin dal principio diede tutto se stesso alla poesia, espresse con slancio giovanile tutte le sue pene interiori, le sue incertezze e le sue esitazioni. Ne nacque un romanzo-confessione di cui voleva fare l'opera della sua vita come Mickiewicz degli Avi. Le Ultime lettere di Jacopo Ortis sono vicinissime nella trama ai Dolori del giovane Werther ${ }^{12}$.

La Wieleżyńska ricorda che Foscolo protestò più volte contro l'idea che la sua opera derivasse da quella dello scrittore tedesco, e negò continuamente di essersene ispirato. Difendeva così il suo romanzo dalle accuse di plagio,

I2. J. Wieleżyńska, Ugo Foscolo — cztowiek, twórca, krytyk, «Przegląd filozoficzny», Warszawa, vol. XXVIII, I925, p. I3I. 
insistendo sul fatto che filosoficamente l'Ortis è più profondo e per questo anche più pessimistico. Vi è inoltre una componente patriottica, assente nel Werther.

La studiosa vorrebbe avvicinare al pubblico polacco il romanzo di Foscolo chiamandolo la «quarta parte degli Avi» (formula che doveva risultare chiara ai lettori polacchi), essa spiega che molti motivi sono affini nelle due opere, anche se diverse le forze psichiche in gioco:

Werther sta nel mezzo, Ortis sulle ali della debolezza, Gustaw sulle ali della forza. Accanto a Ortis Gustaw sembra un giovane Ercole, ma dei tre è lui quello che soffre di più e con più passione, sanguinando. Trasportato in un altro ambiente, Werther potrebbe ricominciare a vivere. Ortis è arrabbiato con tutti, malato, nevrotico. Un tipo femminile, non solo se paragonato a Werther, ma anche a Gustaw, che compie azioni folli, ma ha il fondo dell'anima sano, come lo spirito, né sarebbe capace di scrivere lettere chilometriche come Ortis, analizzando se stesso e il mondo. E poi i personaggi attorno a Gustaw hanno una vera fisionomia, sono vivi, mentre quelli che incontra Ortis son artificiali $[\ldots]^{13}$.

Ma l'aspetto più importante dell'opera, sostiene Wieleżyńska, è l'ispirazione patriottica, che solleva l'Ortis tanto più in alto rispetto al Werther. Gustaw, che vive nel profondo del cuore la tragedia di non poter sposare la donna amata, è destinato a soffrire con la stessa intensità la tragedia della sua patria. Ortis, invece, è capace solo di pianto, passivo com'è non si muove, rimane inadatto all'azione, non ha la forza di servire la patria. E così, mentre Gustaw, vinto l'egoismo del proprio dolore interiore, si avventura negli spazi sconfinati dell'anima, mentre Dante, morta Beatrice, trascende i ristretti confini della Vita nuova e si solleva negli spazi altissimi della Divina Commedia, Foscolo, dato sfogo a quella parte della sua anima che delirando invocava la morte, preferì dar voce a un'altra corda del suo carattere, cioè all'ironia.

Così si conclude l'analisi del romanzo foscoliano. La studiosa osserva che lo sfogo autobiografico non svolse la funzione terapeutica che gli era stata assegnata. Nella sua successiva produzione letteraria, Foscolo sarà tuttavia capace di un dialogo lucido con la vita. Avrà uno sguardo diverso sul mondo, come si vede in Didimo Chierico, anch'esso proiezione autobiografica. Nasceranno inoltre le pagine di un uomo capace di passione: $\mathrm{i}$ sonetti. L'Ortis, malgrado tutto, è una stilizzazione, è letteratura. Alcune frasi, certi pianti, potrebbero fare piangere pure noi. Ma nei sonetti il 
dolore è spontaneo, la commozione non è forzata, l'autore ci si presenta così com'è, senza sentimentalismo eccessivo ${ }^{\mathrm{I4}}$.

I sonetti segnano il passaggio alla poesia più matura, alle Odi. In esse si esprimerebbe l'attaccamento di Foscolo al neoclassicismo, e la studiosa richiama il giudizio di Carducci che le affiancava alle poesie di Parini, mentre Croce le scioglieva dal rimprovero di essere un'imitazione del Settecento, vedendovi «un inno al bello che comunica con i sensi e l'immaginazione del poeta». Nelle Odi si sente il tepore della Grazia, un classicismo sereno e pacato, come il rinascimento del Rinascimento. Dalle Odi è breve la strada che conduce ai capolavori foscoliani, cioè ai Sepolcri e alle Grazie.

La Wieleżyńska riassume il contenuto del carme soffermandosi sui passi in cui Foscolo solleva interrogativi universali, osserva che l'idea del ciclo della natura fu derivata da Vico, punta sui passi che danno rilievo alla memoria storica, e mostra come la fine del carme sia una meditazione omerica sopra le rovine di Troia, simbolo della vita che nella poesia sopravvive a tutto, anche alla fragilità delle vicende umane.

La sezione successiva del saggio è dedicata alle Grazie, che la studiosa chiama «epopea della civiltà». Secondo lei, si tratta di uno degli esiti più alti della lirica foscoliana, e si duole che non sia stato portato a termine. Questa volta, fonte d'ispirazione sono le Grazie di Canova. Ma le Grazie sono soprattutto un simbolo di civiltà, una penetrazione nella sfera dello spirito, un'esaltazione della poesia, concepita come il bello assoluto, capace di competere con tutte le arti figurative.

La Wieleżyńska passa inoltre in rassegna i lavori foscoliani di storia e critica letteraria che lo resero famoso anche in Inghilterra, dove li scrisse, e dove fu apprezzato, fra l'altro, come studioso di Dante. Essa limita però la sua indagine all'Orazione inaugurale recitata a Pavia nel I809, e alle lezioni pubbliche tenute a Londra nel I823.

Principio della critica foscoliana, secondo Wieleżyńska, è un atteggiamento antimetafisico, una filosofia della ragione (in polacco «zdrowy rozsądek» che significa «il ragionamento della mente sana»), che in sociologia guarda a Hobbes e in politica a Machiavelli. Secondo Foscolo, il genio va di pari passo con i bisogni vitali, e la lingua è necessaria per esprimere tutto ciò che crea l'immaginazione. La poesia è quindi la lingua di una nazione, e la storia della letteratura di una nazione è praticamente la storia della sua lingua. Questo è il principio secondo cui il poeta tratteggiò al pubblico inglese la storia della letteratura italiana. Foscolo sostenne poi 
anche che l'italiano aveva subito pochissimi cambiamenti dai tempi di Dante a oggi, e questo spiega perché l'italiano antico (a differenza del francese o dell'inglese antico) sia comprensibile per il lettore dei nostri tempi. La Wieleżyńska ricorda che Foscolo, tra gli autori antichi, ammirava Pier della Vigna, Dante e Petrarca, mentre trovava piatto e povero d'immaginazione lo stile del Decamerone. Questi lavori di Foscolo erano di particolare interesse per il pubblico polacco, a causa del suggerito nesso fra lingua e letteratura.

La studiosa polemizza, nel seguito del suo ampio saggio, con le riflessioni foscoliane su Dante e Petrarca, che rappresenterebbero, secondo il poeta, due poli opposti non solo di letteratura, ma anche di storia e di civiltà. In questa tesi si riflette secondo lei l'anima «bipolare» di Foscolo, divisa fra la passione che emana dai sonetti e «l'azzurro ideale» delle Grazie. Wieleżyńska cerca inoltre di chiarire l'atteggiamento di Foscolo verso il romanticismo, e spiega il suo allontanamento col fatto che dopo essere stato a lungo affascinato dalla poesia ossianica, era giunto alla conclusione che le sue traduzioni potevano impedire il genuino sviluppo della letteratura italiana, il cui romanticismo fu infatti molto atipico rispetto a quello degli altri paesi europei.

Diremo, in conclusione, che il lungo saggio della Wieleżyńska-Dickstein mirava più a fare conoscere e apprezzare Foscolo come poeta che come narratore. Alcune sue riflessioni potranno sembrarci oggi superate, ma sarebbe difficile negare che essa sia stata la maggiore studiosa polacca della cultura italiana nel periodo compreso fra le due guerre mondiali.

Nel supplemento «Letteratura e arte» del quotidiano «Dziennik Poznański», nella rubrica Necrologi si può leggere nel I928:

In Italia si celebra il centenario della morte di Ugo Foscolo, veneziano, poeta, drammaturgo e critico letterario. Questo fervente patriota, prima entusiasta di Napoleone poi suo nemico, democratico e infuocato nelle sue orazioni, che diede prova del suo patriottismo con le azioni da lui compiute, sta a cavallo, come scrittore, tra neoclassicismo e romanticismo. I suoi drammi, per esempio Aiace e Ricciarda, mostrano un forte influsso di Alfieri e sono opere di scarso valore. Meglio si espresse Foscolo in poesia. Scriveva versi anacreontici, odi oraziane, elegie che attingono a Rousseau. Univa l'eroismo al sentimentalismo. De Sanctis lodò l'ode A Luigia Pallavicini, mentre Carducci parlò del sonetto Alla sera considerandolo, con Dante, Petrarca e alcune poesie di Tasso, uno dei vertici della lirica italiana [...]. I Sepolcri sono il suo poema più noto, di cui ci dice Carducci che per la profondità del pensiero richiama Pindaro. Foscolo è anche l'autore del primo romanzo moderno italiano, Ostatnie listy Jakóba Orti [sic!] e come critico è stato il primo a insistere sul fatto che l'opera d'arte è un fenomeno psicologico. Secondo De Santis [sic!] Ugo Foscolo fu anche il miglior conoscitore di Petrarca. 
Nonostante le molte inesattezze, rimane significativo in questa pagina il desiderio di attirare l'attenzione del lettore di Poznań sulla figura di Foscolo e fornire qualche notizia sulle sue opere e sulla loro ricezione critica in Italia.

\section{Osservazioni conclusive}

In conclusione, è d'uopo ripetere che nella Polonia dell'Ottocento il poeta dei Sepolcri fu meno letto e ebbe meno successo di autori cattolici come Pellico o Manzoni. Solo nella seconda metà del secolo crebbe l'interesse per la sua opera. Foscolo rimase però nell'ombra dell'Alfieri, il cui messaggio politico convinse di più i polacchi. Bisogna anche dire che nella prima metà dell'Ottocento, sia tra gli emigrati che tra i patrioti rimasti in Polonia, era ancora vivo e attuale il mito di Napoleone. Benché il Principato di Varsavia fosse stato un'esperienza simile a quella della Cisalpina, per i polacchi Napoleone restava un genio politico e militare. Le critiche mossegli da Foscolo non rispondevano alle aspettative ideologiche dei lettori polacchi. A questo si aggiunga che Foscolo apparteneva a una generazione di scettici, non a quella dei buoni cristiani.

Era dunque un autore complicato per gli studiosi polacchi: patriota ma antinapoleonico, poeta di valore, ma in cui si scontrano due secoli «l'un contro l'altro armato", e quindi difficile da classificare. Solo in un passato più recente gli orizzonti si sono allargati e col saggio della Wieleżyńska Foscolo è entrato a far parte della famiglia dei poeti italiani conosciuti e amati dai polacchi. Oggi in Polonia lo leggono tutti i numerosi studenti di italianistica e chi s'interessa di letteratura e cultura italiana è perfettamente consapevole del posto che egli occupa nel Pantheon della letteratura italiana. 